\section{Resistance Trends of Bacterial Pathogens Isolated from ICUs of European-Gaza Hospital, Palestine}

\section{Emad Abouelkhair ${ }^{1}$, Nasser Qudaih², Nahed Al Laham ${ }^{3}$}

\author{
1Department of Biological Sciences, Al Azhar University- \\ Gaza, Palestine \\ 2Clinical Laboratories, European-Gaza Hospital, Palestine \\ 3Department of Laboratory Medicine, Faculty of Applied \\ Medical Sciences, Al Azhar University-Gaza, Palestine \\ * Corresponding author \\ e-mail address: e.khair@alazhar.edu.ps
}

https://doi.org/10.33976/IUGNS.30.1/2022/1

\begin{abstract}
:
Background: The emergence and spread of antimicrobial resistance among bacterial pathogens have increased to worrying levels in recent years. Multi-drug resistant (MDR) bacteria are known to cause lifethreatening infections especially in Intensive Care Units (ICUs).

Objectives: To study the type, frequency and resistance trends of bacterial pathogens isolated from the ICU departments of the European Gaza Hospital.

Materials and methods: Prospective cross-sectional study was performed on the patients who were admitted to the ICU departments of the European Gaza Hospital for nine months. All isolated bacterial pathogens were diagnosed phenotypically and biochemically, tested against the most common used antibiotics by disc diffusion method.

Results: Ninety clinically significant isolates obtained from 476 various clinical specimens from ICU patients were included in this study (18.9\%). The isolated organisms include both Gram positive cocci and Gram-negative rods. Most of the isolated pathogens were MDR. The most effective agents against MRSA were vancomycin $(81.8 \%)$ and amikacin (72.7\%). Moreover, in addition, meropenem, amikacin and piperacillin-tazobactam were the most effective antimicrobial agents against Gram negative isolates except Acinetobacter baumannii isolates which exhibited high resistance to all tested antimicrobial agents, including the meropenem, were more than half found to be resistant $(52.3 \%)$.

Conclusion: Most of Enterobacteriaceae members and other Gramnegative rods isolated in this study from the ICU departments of the European-Gaza hospital were found to be MDR.
\end{abstract}

\section{Keywords:}

European Gaza Hospital, ICU departments, MDR, Antimicrobial resistance, Gaza strip 


\section{Introduction:}

The nature of ICU environment makes it a focus for the emergence and spread of MDR pathogens. Critically ill patients in the ICU are 2-5times more likely to acquire a nosocomial infection than those in general wards thus leading to prolong hospital stay and require a significant economic cost. Spreading of MDR pathogens limits the therapeutic option for the patients of the ICU departments therefore new antibiotics are needed for the treatment of such organisms (Doyle et al., 2011). Many antibiotic-resistant bacteria including both Gram-positive and Gram- negative, cause a clinically significant danger of infection among ICU patients.

Nosocomial infections are a significant public health problem, the ICU records an occurrence rate two to five times higher than in other health care services (Vitrat et al., 2014). Thus, it is reported that the risk of contracting bacteremia in ICU can reach $31.5 \%$ to $82.4 \%$ of the patients. It has been estimated that the relation between nosocomial infection and fatal outcomes in critically ill patients could be responsible for 10000 to 20000 deaths per year in France (Haddadi et al., 2013). The aim of this study is to isolate and characterize the causative pathogenic bacteria from the ICUs departments of the European Gaza hospital (EGH) and to evaluate its resistance pattern to the most common used antibiotics.

\section{Materials and methods}

\section{Study setting and sampling}

This prospective cross-sectional study was performed on patients who were admitted to the ICU departments of the EGH. All patients who occupied a bed in the participating ICU departments of the EGH including adult ICU (AICU), pediatric (PICU), Special Care Baby Unit (SCBU) and Coronary care unit (CCU) during the study period. Interview of the patient or guardian was used for filling a simple questionnaire, which designed for matching the study need. The questionnaire included questions on socio-demographic data (age, sex, education, history, smoking and employment status). The necessary approvals to conduct this study were obtained from the department of biological sciences at Al- Azhar University-Gaza and from Palestinian Ministry of Health $(\mathrm{MOH})$. Moreover, ethical approval from the local Helsinki committee at the ministry of health in Gaza strip (Approval no. PHRC/HC/19/15) was obtained for performing the current study.

\section{Antimicrobials used}

Penicillin G (10 U), ampicillin (10 $\mu \mathrm{g})$, amoxicillin (30 $\mu \mathrm{g})$, cefuroxime (30 $\mu \mathrm{g})$, ciprofloxacin $(5$ $\mu \mathrm{g})$, ceftazidime $(30 \mu \mathrm{g})$, gentamicin $(10 \mu \mathrm{g})$, oxacillin $(1 \mu \mathrm{g})$, vancomycin $(30 \mu \mathrm{g})$, 
piperacillin/tazobactam $(100 / 10 \mu \mathrm{g})$, cephalothin $(30 \mu \mathrm{g})$, tobramycin $(10 \mu \mathrm{g})$, meropenem $(10 \mu \mathrm{g})$, ceftriaxone $(30 \mu \mathrm{g})$, amikacin $(30 \mu \mathrm{g})$, piperacillin $(100 \mu \mathrm{g})$ and trimethoprim/sulfamethoxazole $(25 / 23.75 \mu \mathrm{g})$.

\section{Study location}

The study was conducted at the microbiology unit of the clinical laboratory of the European-Gaza hospital and the microbiology laboratory at the biological sciences department of Al Azhar university-Gaza.

\section{Microbiological methods}

\section{Sample collection}

Samples including urine, sputum, folly catheters, nasogastric tubes, endotracheal, ascites fluid, CSF and others were collected by the attending physician at the ICU departments in sterile containers. Other samples like pus and infected wound samples were collected with sterile cotton swabs.

Samples were delivered immediately to the medical microbiology laboratory at EGH after collection and cultured on MacConkey agar, Blood agar, Chocolate agar, and thioglycolate agar and incubated at $37^{\circ} \mathrm{C}$ for $24-48 \mathrm{hrs}$.

\section{Sampling duration and sample size}

Different samples were collected from patient who were admitted to the ICU departments over the period of nine months (May-June, 2015). A total of 472 different samples were collected, 387 samples were negative (no bacterial growth) and 90 samples were positive as shown in table 1.

Table (1): Microbiological samples from the ICUs departments

\begin{tabular}{|l|c|c|c|c|}
\hline \multicolumn{1}{|c|}{ Sample type } & AICU & PICU & SCBU & CCU \\
\hline Blood & 1 & 4 & 22 & 0 \\
\hline Sputum & 21 & 10 & 11 & 0 \\
\hline Swab (PUS) & 6 & 1 & 4 & 3 \\
\hline Urine & 1 & 0 & 3 & 0 \\
\hline Fluid & 0 & 0 & 1 & 0 \\
\hline CSF & 0 & 0 & 1 & 4 \\
\hline Total & 29 & 15 & 42 & 1 \\
\hline
\end{tabular}

AICU, adult intensive care unit; PICU, pediatric intensive care unit; SCBU, special baby care Unit; CCU, coronary care unit 


\section{Culture and identification of causative pathogens}

Specimens were directly inoculated onto blood, chocolate, and MacConkey agar plates. All plates were incubated for 24-48 hours aerobically at 35-37 ${ }^{\circ} \mathrm{C}$ with the exception of chocolate agar plates that were incubated in a $\mathrm{CO}_{2}$ incubator to achieve a capnophilic condition with $5 \% \mathrm{CO}_{2}$ that is suitable condition for the isolation of fastidious pathogenic bacteria such as Streptococcus pneumoniae and Haemophilus influenzae.

Colonies were identified according to standard microbiological methods including culture characteristics, colony morphology, mucus production, color, pigment production, sugar fermentation, hemolysis on blood agar and other different characteristics. Gram staining, Catalase test, Coagulase test, Oxidase test, Indole test, Methyl red test, Voges-Proskauer test, Citrate utilization test, and Urease test were performed. Positive cultures growing on both Blood and MacConkey agar plates were submitted to the biochemical identification API20E system (Anon, 1991).

\section{Antimicrobial susceptibility test (The Kirby-Bauer method)}

The isolates were tested for antimicrobial susceptibility by Kirby-Bauer disk diffusion method on Mueller-Hinton agar (MHA) (HiMedia, India). The inoculated plates were incubated overnight at $37{ }^{\circ} \mathrm{C}$, and the zone of inhibition of bacterial growth was used as a measure of susceptibility. Performing the test and interpretation of results was done according to the criteria of the Clinical and Laboratory Standards Institute (CLSI, 2011). The isolates that showed resistant to at least one antibiotic in three or more antimicrobial classes were categorized as MDR, and if they were resistant to at least one agent in all but equal or less than two classes, they were identified as XDR (Magiorakos et al., 2012). Methicillin resistance was detected using oxacillin (1 $\mu \mathrm{g})$ disc diffusion test. Plates with oxacillin were incubated at $33-35{ }^{\circ} \mathrm{C}$ because testing at temperatures above $35{ }^{\circ} \mathrm{C}$ may not detect MRSA as recommended by CLSI guidelines (CLSI, 2011). Zone of inhibition for each antimicrobial agent was interpreted, reporting the organism as resistant, intermediate or susceptible. S. aureus ATCC 33592 (mecA positive, resistant) and S. aureus ATCC 29213 (mecA negative, susceptible) were used as quality control strains.

\section{Statistical analyses}

Obtained data were analyzed statistically using SPSS program (SPSS version, 22). Frequencies, cross tabulation and appropriate statistical tests as Chi-square test, fisher exact test and others were performed. 


\section{Results}

\section{Demographic characteristics of the study population}

A total of 1300 patients were admitted to the ICU departments in the EGH during the study period, 476 different samples were collected. Only 90 (18.9\%) samples were positive while 386 samples were negative. The patients aged between 1 month and 93 years, with mean age of 16.97 years.

The distribution of the study participants showed that $49(54.4 \%)$ were male and $41(45.6 \%)$ were female; 68 (75.6\%) were not married (single, widowed and divorced) and 22 (24.4\%) were married; $40(66.7 \%)$ patients were not educated, $8(8.9 \%)$ were in primary school, $12(13.3 \%)$ were in secondary school and $10(11.1 \%)$ had university education; $10(11.1 \%)$ of the study participants were employed and 80 (88.9\%) were unemployed; the majority of study participants $88(97.8 \%)$ were non-smokers and $2(2.2 \%)$ were smokers.

The mortality rate among the patients of the ICU departments who have positive culture was $34 \%$ (31/90). Their resistance profile showed that tigecycline, amikacin, piperacillin/tazobactam were the most effective antibiotics respectively. Their pathogens were completely resistant to ampicillin and amoxicillin. Moreover, they were almost resistant to ceftriaxone, cefuroxime, cephalothin, trimethoprim/sulfamethoxazole, tobramycin and ciprofloxacin (Figure 1).

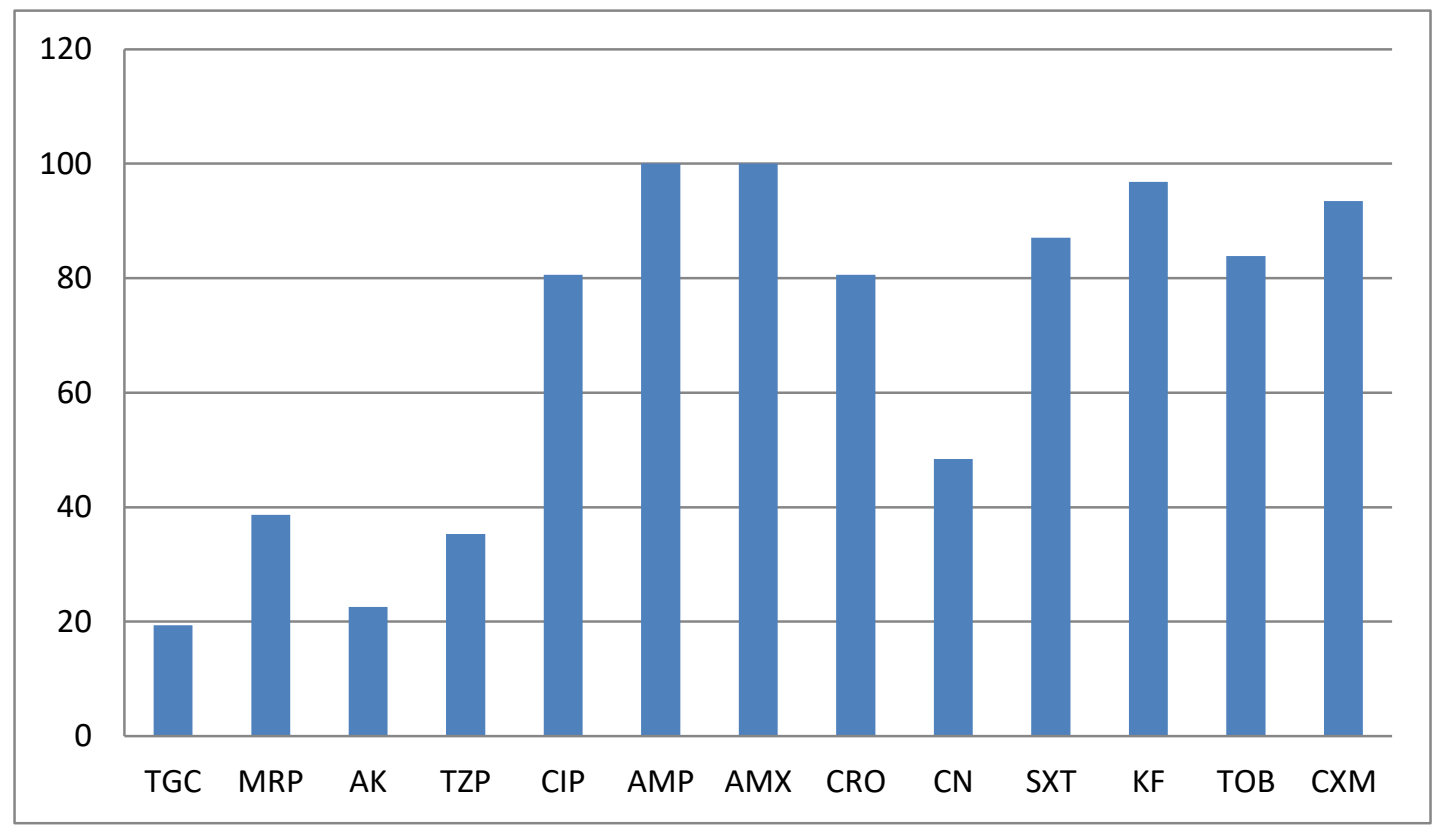

Figure (1): Resistance profile of isolated pathogens recovered from died patients while they alive

Abbreviations: AK, amikacin; AMP, ampicillin; AMX, amoxicillin; CIP, ciprofloxacin; CN, gentamicin; CRO, ceftriaxone; CXM, cefuroxime; MRP, meropenem; SXT, trimethoprim/sulfamethoxazole; TOB, tobramycin; TZP, piperacillin-tazobactam; KF, cephalothin; TGC, Tigecycline. 


\section{Isolated pathogens}

Ninety clinical isolates were recovered in this study and included Gram positive and negative isolates.

\section{Gram-positive isolates}

The total of Gram-positive isolates was 15: Eleven methicillin resistance Staphylococcus aureus (MRSA) isolates were recovered from $\operatorname{AICU}(n=4), \operatorname{PICU}(n=2)$ and $\operatorname{SCBU}(n=5)$. While, four Streptococcus spp. isolates were recovered from SCBU $(n=3)$ and PICU $(n=1)$.

\section{Gram-negative isolates}

As shown in (table 2), there were 75 Gram negative isolates recovered in this study $(\mathrm{n}=75)$. There were ten genera of bacterial pathogens recovered where Klebsiella pneumoniae, Acinetobacter baumannii and Pseudomonas aeruginosa were the highest recovered pathogens and isolated mostly from the special baby care unit.

Figure (2) shows the resistance profile of all isolated pathogens to the tested antibiotics. Most of the isolates showed multiple resistance to these tested antibiotics.

Table (2): Distribution of Gram-negative isolated pathogen according to the ICU department

\begin{tabular}{|l|c|c|c|c|c|}
\hline \multirow{2}{*}{ Isolated pathogen } & \multicolumn{4}{|c|}{ ICU department } & \multirow{2}{*}{$\begin{array}{c}\text { Total } \\
(\%)\end{array}$} \\
\cline { 2 - 5 } & AICU & PICU & SCBU & CCU & \\
\cline { 2 - 5 } & No. & No. & No. & No. & \\
\hline E. coli & 2 & 1 & 1 & 0 & $4(5.3)$ \\
\hline P. aeruginosa & 5 & 4 & 6 & 1 & $16(21.3)$ \\
\hline Alcaligenes spp. & 0 & 1 & 0 & 0 & $1(1.3)$ \\
\hline K. pneumoniae & 8 & 3 & 11 & 2 & $24(32.0)$ \\
\hline A. baumannii & 7 & 2 & 12 & 0 & $21(28)$ \\
\hline Enterobacter cloacae & 1 & 1 & 0 & 0 & $2(2.66)$ \\
\hline $\boldsymbol{P}$. mirabilis & 1 & 0 & 1 & 0 & $2(2.66)$ \\
\hline Morganella morganii & 0 & 0 & 2 & 0 & $2(2.66)$ \\
\hline K. oxytoca & 1 & 0 & 0 & 1 & $2(2.66)$ \\
\hline Serratia spp. & 0 & 0 & 1 & 0 & $1(1.3)$ \\
\hline & Total & & & & $75(100 \%)$ \\
\hline
\end{tabular}




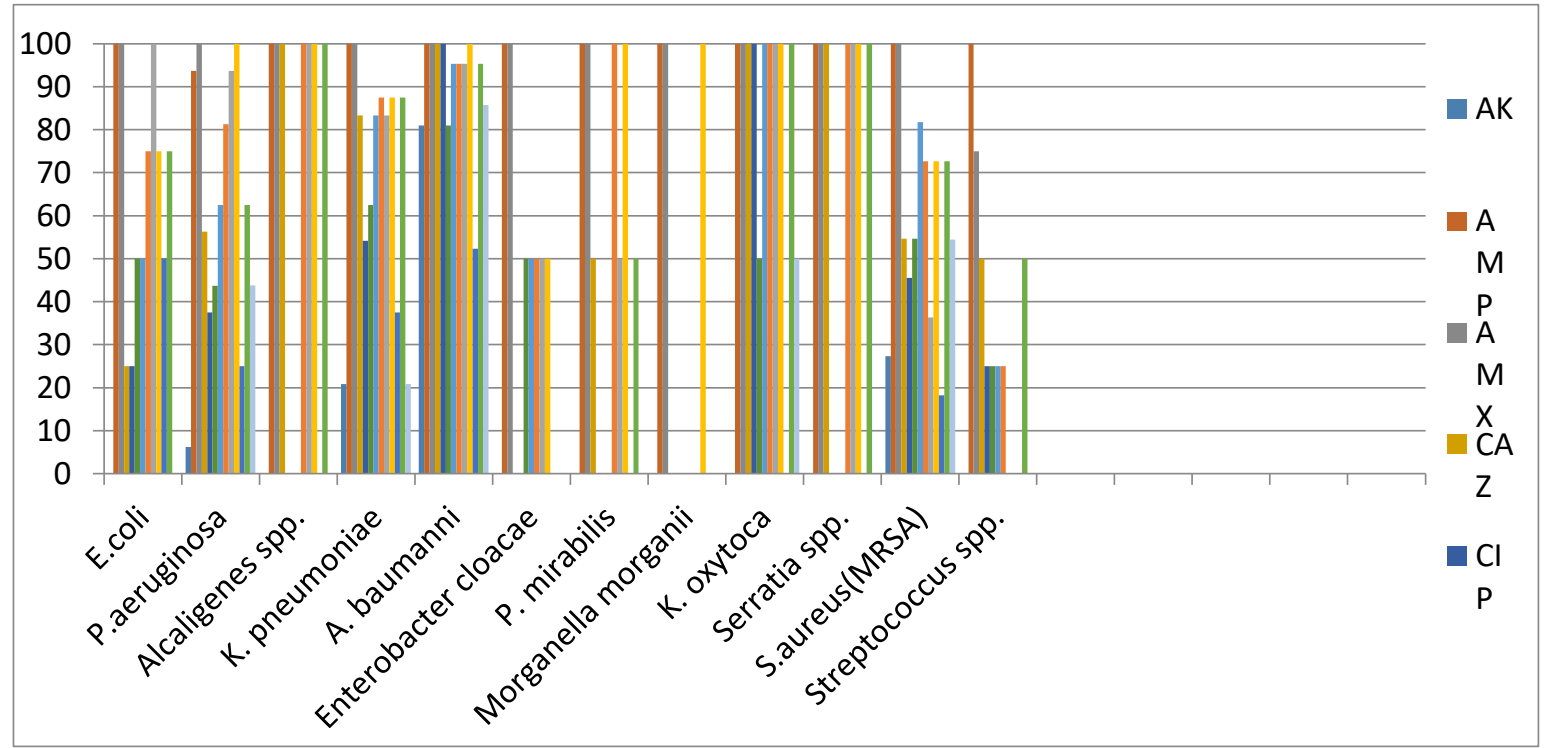

Figure (2): Resistant profile of all isolated pathogens to the tested antibiotics

\section{Resistance profile of Gram-positive isolates to different classes of antimicrobial agents}

From table (3), almost all MRSA isolates (81.8\%) were MDR (resistant to at least one agent in three or more antimicrobial categories) except for (18.2\%). Moreover, all the isolates were resistant to amoxicillin, ampicillin, oxacillin, and penicillin.

Table (3): Antibiogram resistance typing of 11 MRSA isolates

\begin{tabular}{lcc}
\hline Resistance pattern & Resistance type & No. of isolates (\%) \\
AMP, AMX, P & I. & $1(9.1)$ \\
AMP, AMX, P, CRO, CXM & II. & $1(9.1)$ \\
AMP, AMX, P, CRO, CN, TOB & III. & $2(18.2)$ \\
AMP, AMX, P, CRO, CAZ, KF, CXM, DA & IV. & $1(9.1$ \\
AMP, AMX, P, TZP, KF, TOB, CXM, DA, & V. & $1(9.1)$ \\
AMP, AMX, P, TZP, CAZ, CIP, CRO, KF, TOB, CXM, SXT, DA & VI. & $1(9.1)$ \\
AMP, AMX, P, TZP, CAZ, CIP, CRO, AK, CN, KF, TOB, CXM, DA & VII. \\
AMP, AMX, P, TZP, CAZ, CIP, CRO, CN, KF, TOB, CXM, DA, & VIII. \\
MRP, AK & & $2(18.2)$ \\
\end{tabular}

Abbreviations: AK, amikacin; AMP, ampicillin; AMX, amoxicillin; CAZ, ceftazidime; CIP, ciprofloxacin; CN, gentamicin; CRO, ceftriaxone; CXM, cefuroxime; DA, clindamycin; MRP, meropenem; SXT, trimethoprim/sulfamethoxazole; $\mathrm{P}$, penicillin; TOB, tobramycin; TZP, piperacillin-tazobactam; KF, cephalothin. 


\section{Resistance profile of Gram-negative isolates to different classes of antimicrobial agents}

Table 4 showing that $E$. coli $(n=4)$ were susceptible to amikacin and piperacillin-tazobactam. Moreover, $50 \%$ of $E$. coli isolates were susceptible to meropenem. Among P. aeruginosa $(\mathrm{n}=16)$, meropenem and amikacin were the most effective antimicrobial agents. Among Alcaligenes spp., meropenem, amikacin, piperacillin-tazobactam, ciprofloxacin, ceftriaxone and gentamicin were the most effective drugs. Among $K$. pneumoniae isolates $(n=24)$, Amikacin and piperacillintazobactam were the most effective agents, while meropenem resistance was encountered in 9/24 (37.5\%) of the isolates. In the case of A. baumannii $(\mathrm{n}=21)$, amikacin, piperacillin-tazobactam and gentamicin showed poor activity with only few isolates being susceptible. Meropenem resistance was encountered in 11/21(52.4\%) isolates. Enterobacter cloacae $(\mathrm{n}=2)$, were susceptible to meropenem, amikacin, piperacillin-tazobactam, ceftazidime, ciprofloxacin and tobramycin. Among P. mirabilis (n=2), piperacillin-tazobactam, gentamicin, ciprofloxacin, amikacin, meropenem and ceftriaxone all were $100 \%$ effective. The two Morganella morganii isolates were susceptible to all tested antibiotics except for ampicillin, amoxicillin and cephalothin. Both isolates of $K$. oxytoca were $100 \%$ susceptible to amikacin and meropenem. The isolate of Serratia spp., was sensitive to meropenem, ciprofloxacin, ceftriaxone, amikacin, piperacillin-tazobactam, and gentamicin.

Table (4): Susceptibility profile of Gram-negative isolates to other classes of antimicrobial agents

\begin{tabular}{|c|c|c|c|c|c|c|c|c|c|c|c|}
\hline & 䒿 & 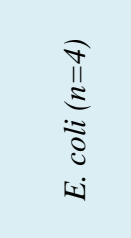 & 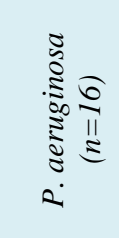 & 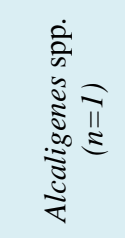 & 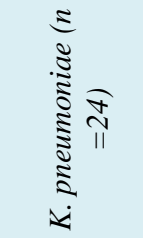 & 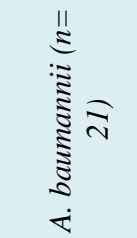 & 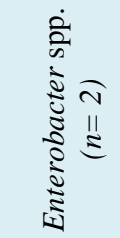 & 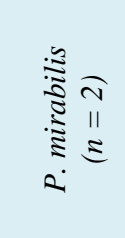 & 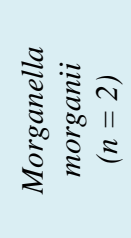 & 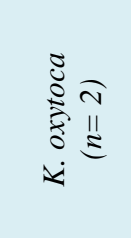 & 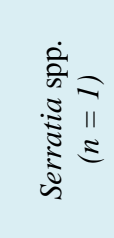 \\
\hline \multirow{3}{*}{ AK } & $\mathbf{S}$ & $4(100.0)$ & $14(87.5)$ & $1(100.0)$ & $19(79.2)$ & $4(19.0)$ & $2(100.0)$ & $1(50.0)$ & $2(100.0)$ & $2(100.0)$ & $1(100.0)$ \\
\hline & I & $0(0.0)$ & $1(6.25)$ & $0(0.0)$ & $0(0.0)$ & $0(0.0)$ & $0(0.0)$ & $1(50.0)$ & $0(0.0)$ & $0(0.0)$ & $0(0.0)$ \\
\hline & $\mathbf{R}$ & $0(0.0)$ & $1(6.25)$ & $0(0.0)$ & $5(20.8)$ & $17(81.0)$ & $0(0.0)$ & $0(0.0)$ & $0(0.0)$ & $0(0.0)$ & $0(0.0)$ \\
\hline \multirow{3}{*}{ AMP } & $\mathbf{S}$ & $0(0.0)$ & $1(6.3)$ & $0(0.0)$ & $0(0.0)$ & $0(0.0)$ & $0(0.0)$ & $0(0.0)$ & $0(0.0)$ & $0(0.0)$ & $0(0.0)$ \\
\hline & I & $0(0.0)$ & $0(0.0)$ & $0(0.0)$ & $0(0.0)$ & $0(0.0)$ & $0(0.0)$ & $0(0.0)$ & $0(0.0)$ & $0(0.0)$ & $0(0.0)$ \\
\hline & $\mathbf{R}$ & $4(100.0)$ & $15(93.7)$ & $1(100)$ & $24(100.0)$ & $21(100.0)$ & $2(100.0)$ & $2(100.0)$ & $2(100.0)$ & $2(100.0)$ & $1(100.0)$ \\
\hline \multirow[b]{3}{*}{ AMX } & $\mathbf{S}$ & $0(0.0)$ & $0(0.0)$ & $0(0.0)$ & $0(0.0)$ & $0(0.0)$ & $0(0.0)$ & $0(0.0)$ & $0(0.0)$ & $0(0.0)$ & $0(0.0)$ \\
\hline & I & $0(0.0)$ & $0(0.0)$ & $0(0.0)$ & $0(0.0)$ & $0(0.0)$ & $0(0.0)$ & $0(0.0)$ & $0(0.0)$ & $0(0.0)$ & $0(0.0)$ \\
\hline & $\mathbf{R}$ & $4(100.0)$ & $16(100)$ & $1(100.0)$ & $24(100.0)$ & $21(100.0)$ & $2(100.0)$ & $2(100.0)$ & $2(100.0)$ & $2(100.0)$ & $1(100.0)$ \\
\hline \multirow{3}{*}{ CAZ } & $\mathbf{S}$ & $3(75.0)$ & $7(43.7)$ & $0(0.0)$ & $3(12.5)$ & $0(0.0)$ & $2(100.0)$ & $1(50.0)$ & $2(100.0)$ & $0(0.0)$ & $0(0.0)$ \\
\hline & I & $0(0.0)$ & $0(0.0)$ & $0(0.0)$ & $1(4.2)$ & $0(0.0)$ & $0(0.0)$ & $0(0.0)$ & $0(0.0)$ & $0(0.0)$ & $0(0.0)$ \\
\hline & $\mathbf{R}$ & $1(25.0)$ & $9(56.3)$ & $1(100.0)$ & $20(83.3)$ & $21(100.0)$ & $0(0.0)$ & $1(50.0)$ & $0(0.0)$ & $2(100.0)$ & $1(100.0)$ \\
\hline
\end{tabular}


Table (4): Continue

\begin{tabular}{|c|c|c|c|c|c|c|c|c|c|c|c|}
\hline \multirow{3}{*}{ CIP } & $\mathbf{S}$ & $2(50.0)$ & $10(62.5)$ & 1(100.0) & $10(41.6)$ & $0(0.0)$ & $2(100.0)$ & $2(100.0)$ & $1(50.0)$ & $0(0.0)$ & $0(0.0)$ \\
\hline & I & $1(25.0)$ & $0(0.0)$ & $0(0.0)$ & $1(4.2)$ & $0(0.0)$ & $0(0.0)$ & $0(0.0)$ & $1(50.0)$ & $0(0.0)$ & $1(100.0)$ \\
\hline & $\mathbf{R}$ & $1(25.0)$ & $6(37.5)$ & $0(0.0)$ & $13(54.2)$ & $21(100.0)$ & $0(0.0)$ & $0(0.0)$ & $0(0.0)$ & $2(100.0)$ & $0(0.0)$ \\
\hline \multirow{3}{*}{$\mathrm{CN}$} & $\mathbf{S}$ & $2(50.0)$ & $10(62.5)$ & $1(100.0)$ & $8(33.3)$ & $3(14.3)$ & $1(50.0)$ & $2(100.0)$ & $2(100.0)$ & $0(0.0)$ & $1(100.0)$ \\
\hline & I & $0(0.0)$ & $0(0.0)$ & $0(0.0)$ & $1(4.2)$ & $1(4.7)$ & $0(0.0)$ & $0(0.0)$ & $0(0.0)$ & $1(50.0)$ & $0(0.0)$ \\
\hline & $\mathbf{R}$ & $2(50.0)$ & $6(37.5)$ & $0(0.0)$ & $15(62.5)$ & $17(81.0)$ & $1(50.0)$ & $0(0.0)$ & $0(0.0)$ & $1(50.0)$ & $0(0.0)$ \\
\hline \multirow{3}{*}{ CRO } & $\mathbf{S}$ & $2(50.0)$ & $6(37.5)$ & $1(100.0)$ & $4(16.7)$ & $1(4.7)$ & $1(50.0)$ & $2(100.0)$ & $2(100.0)$ & $0(0.0)$ & $1(100.0)$ \\
\hline & I & $0(0.0)$ & $0(0.0)$ & $0(0.0)$ & $0(0.0)$ & $0(0.0)$ & $0(0.0)$ & $0(0.0)$ & $0(0.0)$ & $0(0.0)$ & $0(0.0)$ \\
\hline & $\mathbf{R}$ & $2(50.0)$ & $10(62.5)$ & $0(0.0)$ & $20(83.3)$ & $20(95.3)$ & $1(50.0)$ & $0(0.0)$ & $0(0.0)$ & $2(100.0)$ & $0(0.0)$ \\
\hline \multirow{3}{*}{ CXM } & $\mathbf{S}$ & $1(25.0)$ & $3(18.7)$ & $0(0.0)$ & $3(12.5)$ & $1(4.7)$ & $0(0.0)$ & $0(0.0)$ & $2(100.0)$ & $0(0.0)$ & $0(0.0)$ \\
\hline & I & $0(0.0)$ & $0(0.0)$ & $0(0.0)$ & $0(0.0)$ & $0(0.0)$ & $1(50.0)$ & $0(0.0)$ & $0(0.0)$ & $0(0.0)$ & $0(0.0)$ \\
\hline & $\mathbf{R}$ & $3(75.0)$ & $13(81.3)$ & $1(100.0)$ & $21(87.5)$ & $20(95.3)$ & $1(50.0)$ & $2(100.0)$ & $0(0.0)$ & $2(100.0)$ & $1(100.0)$ \\
\hline \multirow{3}{*}{ SXT } & $\mathbf{S}$ & $0(0.0)$ & $1(6.3)$ & $0(0.0)$ & $3(12.5)$ & $1(4.7)$ & $1(50.0)$ & $1(50.0)$ & $2(100.0)$ & $0(0.0)$ & $0(0.0)$ \\
\hline & I & $0(0.0)$ & $0(0.0)$ & $0(0.0)$ & $1(4.2)$ & $0(0.0)$ & $0(0.0)$ & $0(0.0)$ & $0(0.0)$ & $0(0.0)$ & $0(0.0)$ \\
\hline & $\mathbf{R}$ & $4(100.0)$ & $15(93.7)$ & $1(100.0)$ & $20(83.3)$ & $20(95.3)$ & $1(50.0)$ & $1(50.0)$ & $0(0.0)$ & $2(100.0)$ & $1(100.0)$ \\
\hline
\end{tabular}

Table (4): Continue

\begin{tabular}{|c|c|c|c|c|c|c|c|c|c|c|c|}
\hline \multirow{3}{*}{ KF } & $\mathbf{S}$ & $1(25.0)$ & $0(0.0)$ & $0(0.0)$ & $3(12.5)$ & $0(0.0)$ & $1(50.0)$ & $0(0.0)$ & $0(0.0)$ & $0(0.0)$ & $0(0.0)$ \\
\hline & I & $0(0.0)$ & $0(0.0)$ & $0(0.0)$ & $0(0.0)$ & $0(0.0)$ & $0(0.0)$ & $0(0.0)$ & $0(0.0)$ & $0(0.0)$ & $0(0.0)$ \\
\hline & $\mathbf{R}$ & $3(75.0)$ & $16(100.0)$ & $1(100.0)$ & $21(87.5)$ & $21(100.0)$ & $1(50.0)$ & $2(100.0)$ & $2(100.0)$ & $2(100.0)$ & $1(100.0)$ \\
\hline \multirow{3}{*}{ MRP } & $\mathbf{S}$ & $2(50.0)$ & $12(75.0)$ & $1(100.0)$ & $15(62.5)$ & $8(38.2)$ & $2(100.0)$ & $1(50.0)$ & $1(50.0)$ & $2(100.0)$ & $1(100.0)$ \\
\hline & I & $0(0.0)$ & $0(0.0)$ & $0(0.0)$ & $0(0.0)$ & $2(9.5)$ & $0(0.0)$ & $1(50.0)$ & $1(50.0)$ & $0(0.0)$ & $0(0.0)$ \\
\hline & $\mathbf{R}$ & $2(50.0)$ & $4(25.0)$ & $0(0.0)$ & $9(37.5)$ & $11(52.3)$ & $0(0.0)$ & $0(0.0)$ & $0(0.0)$ & $0(0.0)$ & $0(0.0)$ \\
\hline \multirow{3}{*}{ TOB } & $\mathbf{S}$ & $1(25.0)$ & $4(25.0)$ & $0(0.0)$ & $3(12.5)$ & $1(4.7)$ & $2(100.0)$ & $1(50.0)$ & $2(100.0)$ & $0(0.0)$ & $0(0.0)$ \\
\hline & $\mathbf{I}$ & $0(0.0)$ & $2(50.0)$ & $0(0.0)$ & $0(0.0)$ & $0(0.0)$ & $0(0.0)$ & $0(0.0)$ & $0(0.0)$ & $0(0.0)$ & $0(0.0)$ \\
\hline & $\mathbf{R}$ & $3(75.0)$ & $10(62.5)$ & $1(100.0)$ & $21(87.5)$ & $20(95.3)$ & $0(0.0)$ & $1(50.0)$ & $0(0.0)$ & $2(100.0)$ & $1(100.0)$ \\
\hline \multirow{3}{*}{ TZP } & $\mathbf{S}$ & $4(100.0)$ & $9(56.2)$ & $1(100.0)$ & $19(79.2)$ & $3(14.3)$ & $2(100.0)$ & $2(100.0)$ & $2(100.0)$ & $1(50.0)$ & $1(100.0)$ \\
\hline & I & $0(0.0)$ & $0(0.0)$ & $0(0.0)$ & $0(0.0)$ & $0(0.0)$ & $0(0.0)$ & $0(0.0)$ & $0(0.0)$ & $0(0.0)$ & $0(0.0)$ \\
\hline & $\mathbf{R}$ & $0(0.0)$ & $7(43.8)$ & $0(0.0)$ & $5(20.8)$ & $18(85.7)$ & $0(0.0)$ & $0(0.0)$ & $0(0.0)$ & $1(50.0)$ & $0(0.0)$ \\
\hline
\end{tabular}

Abbreviations: AK, amikacin; AMP, ampicillin; AMX, amoxicillin; CAZ, ceftazidime; CIP, ciprofloxacin; CN, gentamicin; CRO, ceftriaxone; CXM, cefuroxime; KF, cephalothin; MRP, meropenem; SXT, trimethoprim/sulfamethoxazole; TOB, tobramycin; TZP, piperacillin-tazobactam.

Table (5) showing that all A. baumannii isolates were highly resistant to the most common used antibiotics, where $(95.3 \%)$ were considered as XDR pathogens (non-susceptibility to at least one agent in all but two or fewer antimicrobial categories i.e., bacterial isolates remain susceptible to only one or two categories), and the rest (4.76\%) were MDR pathogens. While, table (6) showing that most of $P$. aeruginosa isolates were highly resistant to tigecycline and other commonly used 
antibiotics. All P. aeruginosa isolates are considered as MDR pathogens and most of them are XDR pathogens .

Meanwhile, Table (7) showing resistance pattern of $K$. pneumoniae isolates, where all the isolates had complete resistant to amoxicillin and ampicillin. Yet, most of the isolates were MDR pathogens.

Comparing resistance profile of Gram positive and Gram-negative isolates revealed that Gram negative are more resistant than Gram positive to the tested antibiotics as shown in Figure $\mathbf{3}$ and specially they are significantly more resistant to cephalothin and trimethoprim-sulfamethoxazole $(\mathrm{P}=0.001)$ as represented in Table 8 .

Table (5): Antibiogram resistance typing of 21 A. baumannii isolates

\begin{tabular}{lrcc}
\hline Resistance pattern & $\begin{array}{l}\text { Resistance } \\
\text { type }\end{array}$ & No. of isolates (\%) \\
AMP, AMX, CAZ, CIP, KF, TOB & I. & $1(4.76)$ \\
$\begin{array}{l}\text { AMP, AMX, CRO, CAZ, CIP, SXT, KF, CXM } \\
\text { AMP, AMX, CRO, MRP, CAZ, CIP, CN, SXT, KF, TOB, }\end{array}$ & II. & $1(4.76)$ \\
CXM & III. & $1(4.76)$ \\
AMP, AMX, CRO, TGC, TZP, CAZ, CIP, CN, SXT, KF, TOB, & IV. & $1(4.76)$ \\
CXM & V. & $1(4.76)$ \\
$\begin{array}{l}\text { AMP, AMX, CRO, TGC, TZP, CAZ, CIP, CN, SXT, KF, TOB, } \\
\text { AMP, AMX, CRO, AK, TZP, CAZ, CIP, CN, SXT, KF, TOB, }\end{array}$ & VI. & $6(28.5)$ \\
$\begin{array}{l}\text { CXM } \\
\text { AMP, AMX, CRO, MRP, AK, TZP, CAZ, CIP, CN, SXT, KF, }\end{array}$ & VII. & $5(23.8)$ \\
TOB, CXM & & & \\
AMP, AMX, CRO, TGC, MRP, AK, TZP, CAZ, CIP, CN, & VIII. & $5(23.8)$ \\
SXT, KF, TOB, CXM & &
\end{tabular}

Abbreviations: AK, amikacin; AMP, ampicillin; AMX, amoxicillin; CAZ, ceftazidime; CIP, ciprofloxacin; CN, gentamicin; CRO, ceftriaxone; CXM, cefuroxime; KF, cephalothin; MRP, meropenem; SXT, trimethoprim/sulfamethoxazole; TOB, tobramycin; TZP, piperacillin-tazobactam; TGC, tigecycline.

Table (6): Antibiogram resistance typing of $16 P$. aeruginosa isolates

\begin{tabular}{lcc}
\hline Resistance pattern & $\begin{array}{c}\text { Resistance } \\
\text { type }\end{array}$ & No. of isolates (\%) \\
AMP, AMX, SXT, KF, TOB & I. & $1(6.25)$ \\
AMP, AMX, SXT, KF, TGC & II. & $1(6.25)$ \\
AMP, AMX, SXT, KF, TGC, CXM & III. & $2(12.5)$ \\
\hline
\end{tabular}




\begin{tabular}{lcc}
\hline AMP, AMX, SXT, KF, CN, CXM, TGC, & IV. & $2(12.5)$ \\
AMX, TZP, CRO, KF, TOB, TGC, MRP & V. & $1(6.25)$ \\
AMP, AMX, SXT, KF, TGC, CAZ, CRO, TOB, & VI. & $3(18.75)$ \\
CXM & & $1(6.25)$ \\
AMP, AMX, SXT, TZP, CAZ, CRO, KF, TOB, & VII. & \\
CXM & & $2(12.5)$ \\
AMP, AMX, SXT, CAZ, CIP, CRO, KF, TOB, & VIII. & \\
CXM, TGC & & $1(6.25)$ \\
AMP, AMX, SXT, AK, TZP, CAZ, CIP, CRO, & IX. & \\
KF, TOB, CXM & & $2(12.5)$ \\
AMP, AMX, TZP, CAZ, CIP, CRO, SXT, KF, & X. &
\end{tabular}

Abbreviations: AK, amikacin; AMP, ampicillin; AMX, amoxicillin; CAZ, ceftazidime; CIP, ciprofloxacin; CN, gentamicin; CRO, ceftriaxone; CXM, cefuroxime; KF, cephalothin; MRP, meropenem; SXT, trimethoprim/sulfamethoxazole; TOB, tobramycin; TZP, piperacillin-tazobactam; TGC, tigecycline.

Table (7): Antibiogram resistance typing of $24 \mathrm{~K}$. pneumoniae isolates

\begin{tabular}{lcc}
\hline Resistance pattern & $\begin{array}{c}\text { Resistance } \\
\text { type }\end{array}$ & No. of isolates (\%) \\
AMP, AMX, CN & I. & $1(4.16)$ \\
AMP, AMX, CXM & II. & $1(4.16)$ \\
AMP, AMX, TOB, CAZ & III. & $1(4.16)$ \\
AMP, AMX, SXT, KF, CXM & IV. & $1(4.16)$ \\
AMP, AMX, CRO, CAZ, SXT, KF, TOB, CXM & V. & $2(8.3)$ \\
AMP, AMX, CRO, CAZ, CN, SXT, KF, TOB, & VI. & $3(8.3)$ \\
CXM & & $3(4.16)$ \\
AMP, AMX, CRO, MRP, CN, SXT, KF, TOB, & VII. & $1(8.3)$ \\
CXM & VIII. & $2(8.3)$ \\
AMP, AMX, CRO, CAZ, CIP, CN, SXT, KF, & IX. & $1(4.16)$ \\
TOB, CXM & & \\
AMP, AMX, CRO, CN, MRP, CAZ, CIP, SXT, & X. & \\
AMP, AMX, CRO, CN, TZP, CAZ, CIP, SXT, & & \\
KF, TOB, CXM & &
\end{tabular}

Abbreviations: AK, amikacin; AMP, ampicillin; AMX, amoxicillin; CAZ, ceftazidime; CIP, ciprofloxacin; CN, gentamicin; CRO, ceftriaxone; CXM, cefuroxime; KF, cephalothin; MRP, meropenem; SXT, trimethoprim/sulfamethoxazole; TOB, tobramycin; TZP, piperacillin-tazobactam; TGC, tigecycline. 
Table (8): Correlations between Gram positive and Gram-negative isolates to the tested antibiotics

\begin{tabular}{|c|c|c|c|c|c|}
\hline \multicolumn{2}{|l|}{ Antibiotic } & $\begin{array}{c}\text { Gram } \\
\text { negative }\end{array}$ & $\begin{array}{c}\text { Gram } \\
\text { positive }\end{array}$ & $\begin{array}{c}\text { Chi } \\
\text { square }\end{array}$ & $\begin{array}{c}P \\
\text { value }\end{array}$ \\
\hline \multirow{3}{*}{ Cephalothin } & $\mathbf{S}$ & $5(6.7)$ & $6(40.0)$ & \multirow{3}{*}{18.6} & \multirow{3}{*}{$0.001 *$} \\
\hline & $\mathbf{I}$ & $0(0.0)$ & $1(6.7)$ & & \\
\hline & $\mathbf{R}$ & $70(93.3)$ & $8(53.3)$ & & \\
\hline \multirow{3}{*}{ Meropenem } & $\mathbf{S}$ & $45(60.0)$ & $13(86.7)$ & \multirow{3}{*}{4} & \multirow{3}{*}{0.192} \\
\hline & I & $4(5.3)$ & $0(0.0)$ & & \\
\hline & $\mathbf{R}$ & $26(34.7)$ & $2(13.3)$ & & \\
\hline \multirow{3}{*}{$\begin{array}{l}\text { Trimethoprim } \\
\text { sulfamethoxazole }\end{array}$} & $\mathbf{S}$ & $9(12.0)$ & $11(73.3)$ & \multirow{3}{*}{27.2} & \multirow{3}{*}{$0.001 *$} \\
\hline & I & $1(1.3)$ & $0(0.0)$ & & \\
\hline & $\mathbf{R}$ & $65(86.7)$ & $4(26.7)$ & & \\
\hline \multirow{3}{*}{ Cefuroxime } & $\mathbf{S}$ & $10(13.3)$ & $5(33.3)$ & \multirow{3}{*}{5.58} & \multirow{3}{*}{0.053} \\
\hline & I & $1(1.3)$ & $1(6.7)$ & & \\
\hline & $\mathbf{R}$ & $64(85.3)$ & $9(60.0)$ & & \\
\hline \multirow{3}{*}{ Gentamycin } & $\mathbf{S}$ & $29(38.7)$ & $6(40.0)$ & \multirow{3}{*}{2.22} & \multirow{3}{*}{0.265} \\
\hline & $\mathbf{I}$ & $3(4.0)$ & $2(13.3)$ & & \\
\hline & $\mathbf{R}$ & $43(57.9)$ & $7(46.7)$ & & \\
\hline \multirow{3}{*}{ Ciprofloxacin } & $\mathbf{S}$ & $28(37.3)$ & $8(53.3)$ & \multirow{3}{*}{1.53} & \multirow{3}{*}{0.403} \\
\hline & I & $4(5.3)$ & $1(6.7)$ & & \\
\hline & $\mathbf{R}$ & $43(57.3)$ & $6(40.0)$ & & \\
\hline
\end{tabular}

Table (8): Continue

\begin{tabular}{|c|c|c|c|c|c|}
\hline \multicolumn{2}{|l|}{ Antibiotic } & $\begin{array}{c}\text { Gram } \\
\text { negative }\end{array}$ & $\begin{array}{c}\text { Gram } \\
\text { positive }\end{array}$ & $\begin{array}{l}\text { Chi } \\
\text { squar }\end{array}$ & $P$ value \\
\hline $\begin{array}{l}\text { Piperacillin- } \\
\text { tazobactam }\end{array}$ & $\begin{array}{l}\mathbf{I} \\
\mathbf{R}\end{array}$ & $\begin{array}{c}44(58.7) \\
0(0.0) \\
31(41.3)\end{array}$ & $\begin{array}{c}9(60.0) \\
0(0.0) \\
6(40.0)\end{array}$ & 0.09 & 1.0 \\
\hline Tobramycin & $\begin{array}{l}\mathbf{S} \\
\mathbf{I} \\
\mathbf{R}\end{array}$ & $\begin{array}{c}14(18.7) \\
2(2.7) \\
59(78.7)\end{array}$ & $\begin{array}{c}5(33.3) \\
0(0.0) \\
10(66.7)\end{array}$ & 1.9 & 0.513 \\
\hline Amikacin & $\begin{array}{l}\mathbf{S} \\
\mathbf{I} \\
\mathbf{R}\end{array}$ & $\begin{array}{c}50(66.7) \\
2(2.7) \\
23(30.7)\end{array}$ & $\begin{array}{c}12(80.0) \\
0(0.0) \\
3(20.0)\end{array}$ & 1.21 & 0.685 \\
\hline Ampicillin & $\mathbf{S}$ & $1(1.3)$ & $0(0.0)$ & 0.202 & 1.000 \\
\hline
\end{tabular}




\begin{tabular}{|c|c|c|c|c|c|}
\hline & $\begin{array}{l}\mathbf{I} \\
\mathbf{R}\end{array}$ & $\begin{array}{c}0(0.0) \\
74(98.7)\end{array}$ & $\begin{array}{c}0(0.0) \\
15(100)\end{array}$ & & \\
\hline \multirow{3}{*}{ Amoxicillin } & $\mathbf{S}$ & $0(0.0)$ & $1(6.6)$ & \multirow{3}{*}{5.06} & \multirow{3}{*}{0.167} \\
\hline & I & $0(0.0)$ & $0(0.0)$ & & \\
\hline & $\mathbf{R}$ & 75 (100.0) & $14(93.4)$ & & \\
\hline \multirow{3}{*}{ Ceftriaxone } & $\mathbf{S}$ & $20(26.7)$ & $5(33.3)$ & \multirow{3}{*}{0.277} & \multirow{3}{*}{0.75} \\
\hline & I & $0(0.0)$ & $0(0.0)$ & & \\
\hline & $\mathbf{R}$ & $55(73.3)$ & $10(66.7)$ & & \\
\hline \multirow{3}{*}{ Ceftazidime } & $\mathbf{S}$ & $18(24)$ & $7(46.7)$ & \multirow{3}{*}{3.312} & \multirow{3}{*}{0.257} \\
\hline & I & $1(1.3)$ & $0(0.0)$ & & \\
\hline & $\mathbf{R}$ & $56(74.7)$ & $8(53.3)$ & & \\
\hline
\end{tabular}

*significant at $P$ value $<0.05$.

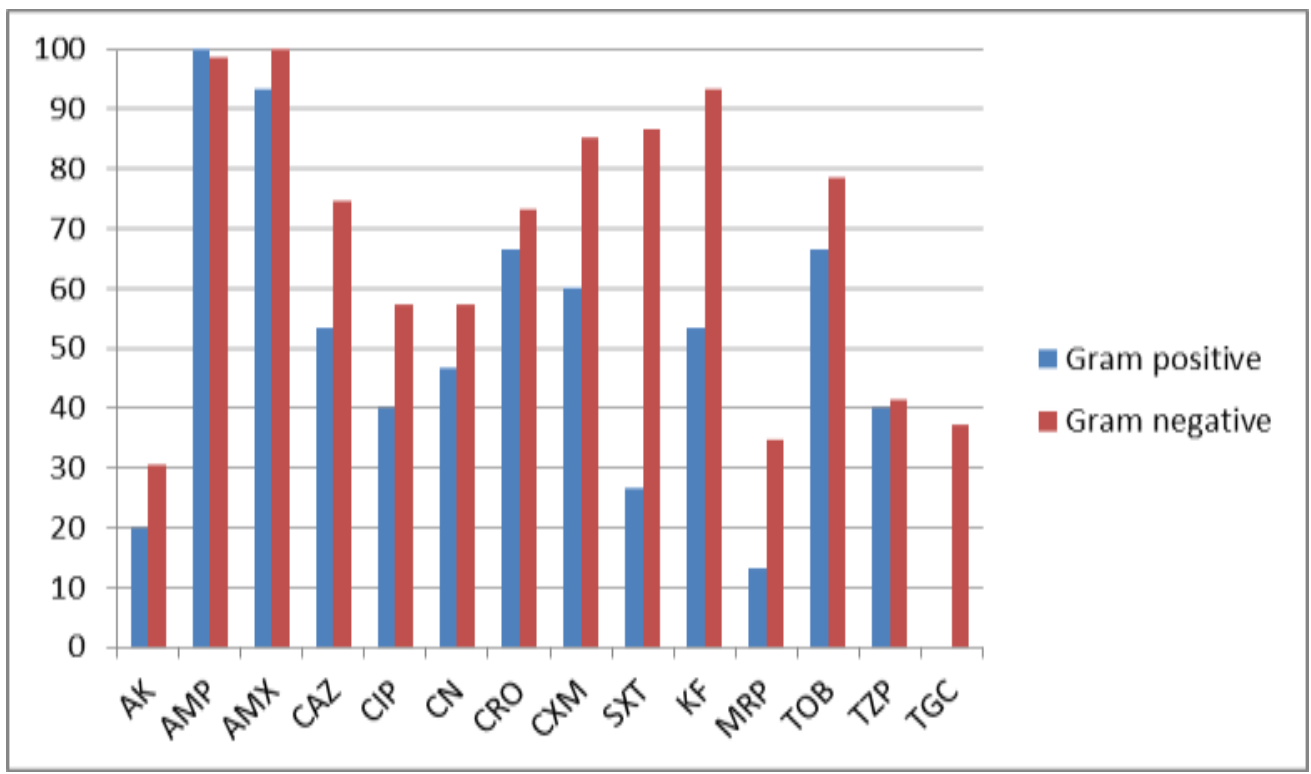

Figure (3): Resistant profile for Gram positive and Gram negative to the tested antibiotics

\section{Discussion}

\section{Prevalence of infection in the ICU departments of EGH}

A total of 476 different clinical samples were collected from four ICU departments of EGH, only 90 samples were culture positive and revealed pathogens. The rate of infection among the patients of the ICUs were (18.9\%). Among the 90 participants who had positive culture, 57(63.3\%) had ICU acquired infection, 21(23.3\%) had hospital acquired infection and 12(13.3\%) had community acquired infection. In this study, we found that the most common sites of infection among the 
ICUs patients were chest infection (ventilator associated pneumonia) (41.1\%), followed by blood infections (31.1\%), wound infections (20.0\%), urinary tract infections (6.7\%) and cerebrospinal fluid infections (1.1\%). This is similar to other previous study whose isolates and their frequency were originated from almost such specimens (Anthony et al., 2008). The rate of nosocomial infection is high in intensive care unit patients, especially for respiratory infections (Toufen et al., 2003). In this study, gram negative bacteria were the predominant pathogens isolated with Klebsiella, Acinetobacter, and Pseudomonas species are the commonest isolates. These pathogens are commonly isolated from patients in ICUs in many studies around the world, however, the prevalence of those pathogens can change over time and settings (Rosenthal et al., 2006; Ding et al., 2009; Doyle et al., 2011; Khanal et al., 2013).

\section{Antimicrobial resistance pattern of isolated bacteria to the commonly used antibiotics}

Since all the isolated pathogens were obtained from the ICU departments where the majority of the patients are critically ill and under heavily antibiotics treatment, this study indicates a high percentage of MDR for the majority of the isolated pathogens. A study conducted in Egypt about the prevalence and risk factors of MRSA, ESBL and MDR bacterial colonization upon admission to an Egyptian medical ICU found that 33\%, 13\%, and 63\% were colonized with ESBL, MDR and MRSA organisms, respectively (Fouda et al., 2016).

MDR is defined as non-susceptibility to at least one agent in three or more antimicrobial categories. XDR is defined as non-susceptibility to at least one agent in all but two or fewer antimicrobial categories (i.e. bacterial isolates remain susceptible to only one or two categories) (Magiorakos et al., 2012). The classes used to define MDR among the Enterobacteriaceae were aminoglycosides (amikacin, tobramycin), $\beta$-lactams (ampicillin, amoxicillin, ceftriaxone, piperacillin-tazobactam), carbapenems (meropenem), fluoroquinolones (ciprofloxacin), glycylcyclines (tigecycline), and sulfonamides (trimethoprim-sulfamethoxazole); the classes used to define MDR A. baumannii were aminoglycosides (amikacin), $\beta$-lactams (ceftazidime, ceftriaxone, or piperacillin-tazobactam), carbapenems (meropenem), fluoroquinolones (ciprofloxacin), and sulfonamides (trimethoprim-sulfamethoxazole); and the classes used to define MDR P. aeruginosa were aminoglycosides (amikacin), $\beta$-lactams (ceftazidime, ceftriaxone or piperacillin-tazobactam), carbapenems (meropenem), and sulfonamides (trimethoprimsulfamethoxazole). 
Eleven isolates of $S$. aureus were collected from the ICU departments and all were MDR. A study was conducted by El-Astal, Z. (2004) found lower resistant rates comparing to the result in this study to penicillin, amoxicillin, ciprofloxacin, ceftriaxone and cefuroxime (El-Astal, Z. 2004). The discrepancy between the results may due to the sample type and size since all the samples in this study were collected from the ICU departments were the most of the pathogens were highly resistant to the most of the antibiotics and the author samples were from other departments. Moreover, the time passed since this study was conducted is about 12 years, where the problem of antimicrobial resistance is growing, exacerbated and worsened worldwide, especially in developing countries including Palestine.

K. pneumoniae isolates were the most abundant among the isolated pathogens. The most effective agents against $K$. pneumoniae isolates was amikacin (79.2\%), piperacillin-tazobactam (79.2\%) and meropenem (62.5\%). Almost all the isolates of $K$. pneumoniae were MDR. The emergence of multidrug resistant $K$. pneumoniae strains has been reported with increasing frequency in several countries worldwide (Pereira et al., 2013). Among $K$. oxytoca, high susceptibility rates were recorded for meropenem (100.0\%), amikacin (100.0\%), while all the isolates of $K$. oxytoca were $100.0 \%$ resistant to ampicillin, amoxicillin, ceftazidime, ceftriaxone, cefuroxime, tobramycin and cephalothin. Hoban et al., in a similar study performed globally found that $K$. oxytoca $(\mathrm{n}=6,940)$ were highly susceptible (>96\%) to amikacin and meropenem (Hoban et al., 2015).

About 52.0\% of A. baumannii isolates were XDR and the rest were MDR. Acinetobacter spp. (particularly A. baumannii) have become resistant to most of the major antimicrobial agents in widespread use, including aminoglycosides, quinolones, $\beta$-lactams, cephalosporins and carbapenems (Vasilev et al., 2008). Acinetobacter spp. have become important pathogens in ICUs, and the development of resistance to carbapenems has been reported worldwide (Nilsson et al., 2011).

Because of its resistance to multiple antimicrobial agents, only few antibiotics found to be effective against $P$. aeruginosa (Fatima et al., 2012). In this study, all the isolates of $P$. aeruginosa were MDR which again carry high risk especially for immunocompromised patients in ICU departments. The highest level of in vitro susceptibility against $P$. aeruginosa in this study was reported for amikacin $(87.5 \%)$ and meropenem $(75.0 \%)$. These results were in agreement with previous study conducted in Gaza strip by Astal Z., 2004 which showed that the 
most effective antimicrobial agents against $P$. aeruginosa were meropenem and amikacin (Astal Z., 2004). Yet, another study found that amikacin was the most effective agent against $P$. aeruginosa (Balode et al., 2013).

All the isolates of $E$. coli in this study were $100 \%$ resistant to ampicillin, amoxicillin and trimethoprim-sulfamethoxazole. Conversely, amikacin and piperacillin/tazobactam were the most effective agents. Carbapenem resistance were detected in two (50.0\%) isolates among E. coli. Shanthi et al., 2011 found that E. coli were most susceptible to amikacin, piperacillintazobactam, and meropenem (Shanthi et al., 2011).

\section{Mortality rate at the ICU departments}

Mortality rate was very high $(34.0 \%)$ among ICUs patients. High mortality rate has been previously reported from ICUs in developing countries (Rosenthal et al., 2006; Rosenthal et al., 2011). This high rate of mortality could be due to the fact that most of the ICU residents are critically ill and they are usually at high risk of gaining nosocomial infections due to their weak immune system. Also, presence of several risk factors such as central lines, urinary catheter, mechanical ventilation and other risk factors, that give higher possibility for gaining an infection by MDR or XDR pathogen is worsening the matter and limited the therapeutic choices which lead finally to death.

\section{Conclusion}

Multiple-drug-resistant Gram-negative bacteria are the most problematic pathogens in the European Gaza hospital, especially in the intensive-care units. The ICU is typically associated with high rates of antibiotic resistance. Few antimicrobial agents remain active against a wide range of organisms. Amikacin and meropenem were the most effective agents against most of the isolated pathogens (except for A. baumannii). A. baumannii considered currently the most troublesome nosocomial pathogen, mainly affecting patients in ICUs. These isolates, widely resistant to the commonly used antimicrobials, have now developed resistance to the carbapenems. 


\section{References}

Anon. (1991): Analytical Profile Index. API 20E. 3rd ed. \# 20/90. BioMérieux S.A. Lyon, France.pp. 420.

Anthony, K. B., Fishman, N. O., Linkin, D. R., Gasink, L. B., Edelstein, P. H. and Lautenbach, E. (2008) Clinical and microbiological outcomes of serious infections with multidrug-resistant gram-negative organisms treated with tigecycline. Clinical infectious diseases, 46(4), pp. 567570.

Astal, Z. (2004) Susceptibility patterns in Pseudomonas aeruginosa causing nosocomial infections. Journal of chemotherapy, 16(3), pp. 264-268.

Balode, A., Punda-Polić, V. and Dowzicky, M. J. (2013) Antimicrobial susceptibility of Gramnegative and Gram-positive bacteria collected from countries in Eastern Europe: results from the Tigecycline Evaluation and Surveillance Trial (TEST) 2004-2010. International journal of antimicrobial agents, 41(6), pp. 527-535.

Bassetti, M., Nicolini, L., Repetto, E., Righi, E., Del Bono, V. and Viscoli, C. (2010) Tigecycline use in serious nosocomial infections: a drug use evaluation. BMC infectious diseases, 10(1), pp. 287-290.

Clinical and Laboratory Standards Institute. Performance Standards for Antimicrobial Susceptibility Testing: Twenty-first Informational Supplement M100-S21. Wayne, PA, USA: CLSI; 2011.

Doyle, J. S., Buising, K. L., Thursky, K. A., Worth, L. J. and Richards, M. J. (2011) Epidemiology of infections acquired in intensive care units. In Seminars in respiratory and critical care medicine, 32(2), pp. 115-138.

European Gaza hospital: Half year annual report, 2011, MOH.

El-Astal, Z. (2004) Bacterial pathogens and their antimicrobial susceptibility in Gaza Strip, Palestine. Pakistan Journal of Medical Sciences, 20(4), pp. 365-370.

Fatima, A., Naqvi, S. B., Khaliq, S. A., Perveen, S. and Jabeen, S. (2012) Antimicrobial susceptibility pattern of clinical isolates of Pseudomonas aeruginosa isolated from patients of lower respiratory tract infections. Springer plus, 1(1), pp. 70-73.

Fouda, R., Soliman, M. S., El Anany, M. G., Abadeer, M. and Soliman, G. (2016) Prevalence and risk factors of MRSA, ESBL and MDR bacterial colonization upon admission to an Egyptian medical ICU. The Journal of Infection in Developing Countries, 10(04), pp. 329-336.

Hoban, D. J., Reinert, R. R., Bouchillon, S. K. and Dowzicky, M. J. (2015) Global in vitro activity of tigecycline and comparator agents: Tigecycline Evaluation and Surveillance Trial 20042013. Annals of clinical microbiology and antimicrobials, 14(1), pp. 1-16.

Khanal, S., Joshi, D. R., Bhatta, D. R., Devkota, U. and Pokhrel, B. M. (2013) $\beta$-lactamaseproducing multidrug-resistant bacterial pathogens from tracheal aspirates of intensive care unit patients at National Institute of Neurological and Allied Sciences, Nepal. International Scholarly Research Notices Microbiology, 2013.

Magiorakos, A. P., Srinivasan, A., Carey, R. B., Carmeli, Y., Falagas, M. E., Giske, C. G. and Paterson, D. L. (2012) Multidrug-resistant, extensively drug-resistant and pandrug-resistant bacteria: an international expert proposal for interim standard definitions for acquired resistance. Clinical Microbiology and Infection, 18(3), pp. 268-281.

Nilsson, L. E., Frimodt-Møller, N., Vaara, M. and Simonsen, G. S. (2011) Comparative activity of tigecycline and tetracycline on Gram-negative and Gram-positive bacteria revealed by a multicentre study in four North European countries. Scandinavian journal of infectious diseases, 43(9), pp. 707-713. 
Pereira, G. H., Garcia, D. O., Mostardeiro, M., Fanti, K. S. and Levin, A. S. (2013) Outbreak of carbapenem-resistant Klebsiella pneumoniae: two-year epidemiologic follow-up in a tertiary hospital. Memórias do Instituto Oswaldo Cruz, 108(1), pp. 113-115.

Rosenthal, V. D., Maki, D. G., Salomao, R., Moreno, C. A., Mehta, Y., Higuera, F. and Leblebicioglu, H. (2006) Device-associated nosocomial infections in 55 intensive care units of 8 developing countries. Annals of internal medicine,145(8), pp. 582-591.

Rosenthal, V. D., Udwadia, F. E., Munoz, H. J., Erben, N., Higuera, F., Abidi, K. and Barnett, A. G. (2011) Time-dependent analysis of extra length of stay and mortality due to ventilatorassociated pneumonia in intensive-care units of ten limited-resources countries: findings of the International Nosocomial Infection Control Consortium (INICC). Epidemiology and infection, 139(11), pp. 1757-1763.

Shanthi, M. and Sekar, U. (2011) In vitro activity of tigecycline against Gram positive and Gramnegative isolates in a tertiary care hospital. Journal of Clinical Diagnostic Research, 5, pp. 1559-1563.

Toufen Junior, C., Hovnanian, A. L. D., Franca, S. A. and Carvalho, C. R. R. (2003) Prevalence rates of infection in intensive care units of a tertiary teaching hospital. Revista do Hospital das Clínicas, 58(5), pp. 254-259.

Vasilev, K., Reshedko, G., Orasan, R., Sanchez, M., Teras, J., Babinchak, T. and Orrico, R. (2008) A Phase 3, open-label, non-comparative study of tigecycline in the treatment of patients with selected serious infections due to resistant Gram-negative organisms including Enterobacter species, Acinetobacter baumannii and Klebsiella pneumoniae. Journal of Antimicrobial Chemotherapy, 62(suppl 1), pp. 129-140.

Vitrat, V., Hautefeuille, S., Janssen, C., Bougon, D., Sirodot, M. and Pagani, L. (2014) Optimizing antimicrobial therapy in critically ill patients. Infection and drug resistance, 7, pp. 261-271. 